\title{
BLOCKCHAIN FOR VEHICLES BASED ON EXONUM PLATFORM
}

\author{
Nurbol Zhumadil, \\ Almaty Management University, Almaty, Kazakhstan, \\ nzhumadil@gmail.com \\ Saltanat Narbayeva, \\ al-Farabi Kazakh National university, Almaty, Kazakhstan \\ narbaevasalta777@gmail.com \\ Timur Bakibayev, \\ Almaty Management University, Almaty, Kazakhstan, \\ timurbakibayev@gmail.com \\ Kuanysh Abeshev, \\ Almaty Management University, Almaty, Kazakhstan, \\ kuanysh.abeshev@gmail.com \\ Kseniya Shubenkova, \\ Kazan Federal University, Russia \\ ksenia.shubenkova@gmail.com
}

DOI: 10.36724/2664-066X-202I-7-I-24-29

\begin{abstract}
We propose a new vehicle monitoring system based on Exonum blockchain platform. This system may help autonomous vehicles to make decisions. This system can also help in investigating crimes and traffic offense. The article describes the main trends in the field of intellectualization of transport systems and mobility. The blockchain technology and its capabilities in enhancing cybersecurity are described through the creation of a safe and reliable system for sending the parameters of the current state of each vehicle through its neighbours. The proposed system will serve as an important step towards the development of a motion control system for connected and autonomous vehicles
\end{abstract}

KEYWORDS: road safety, traffic accident, methods, blockchain, Exonum.

Information about authors

Nurbol A. Zhumadil, master of economics sciences specialty "Management", "Almaty Management University", Almaty, Kazakhstan

Saltanat M. Narbayeva, Ph.D. student specialty "In information security systems", al-Farabi Kazakh National university, Almaty, Kazakhstan

Timur Bakibayev, Ph.D, Professor, Almaty Management University, Almaty, Kazakhstan

Kuanysh Sh. Abeshev, Ph.D. Dean of the Almaty Management University, Almaty, Kazakhstan

Kseniya Shubenkova, Candidate of sciences, Senior lecturer of the "Service of Transport Systems" department, Kazan Federal University, Russia 


\section{Introduction}

The article is devoted to the current security problem, which excludes the intervention of the human factor in forging and altering data. Despite the fact that the blockchain distributed database is one of the most secure in the world, this article additionally considers a number of cases related to data changes to ensure the security of the system. New blockchain-enabled platforms will make it easy to coordinate documents in a shared, distributed ledger, making physical documents unnecessary. Based on the analysis, the proposed system will serve as an important step towards the development of a traffic control system for connected and autonomous vehicles, as well as attracting companies to use Blockchain based on the Exonum platform. This direction further optimizes processes that make it easy to coordinate documents in a shared distributed ledger, which makes physical documents unnecessary.

Intelligent vehicle technology is evolving very quickly, and recent advances suggest that autonomous vehicle navigation will be possible in the near future. At intersections, traffic lights and a stop-light enable drivers to safely navigate the intersections in their vehicle. However, will it make sense in the future for cars with onboard computers "behind the wheel", controls that have been designed with today's human drivers in mind? These advantages, which are being used for intelligent robotic drivers - more precise control, better sensors and less time - we believe that car travel in the future can be safer and easier, but also much more efficient.

In this regard, we believe that absolutely all vehicles will have a full-fledged on-board computer, using secure applications with access to navigation and other sensors in read mode. Therefore, the implementation of blockchain solutions will be quite affordable without additional hardware modifications [1].

\section{Blockchain and transport}

Continuing efforts to resolve the situation indicate that the logistics situation remains problematic. Whether it's blockchain, smart contracts, or escort fees, the industry needs a solution. Without it, shippers and carriers continue to lose money, or at least miss out on opportunities to be more efficient.

Logistics is clearly seen as one of the priority blockchain applications. And here we are talking not only about projects. The giants of the logistics industry are using blockchain to increase their competitiveness. Maersk [4], in collaboration with IBM, launched the TradeLens ecosystem in 2018 for tracking vehicles, containers and cargo, as well as supply chain management. The platform collects and integrates cargo traffic data from industry partners into a single secure blockchain network and provides secure access to information for all stakeholders.

The system allows you to automate almost all document flow and digital business processes associated with transportation logistics, including such operations as obtaining a bill of lading, financial transactions, customs duties and transfer of ownership / responsibility. To do this, TradeLens uses smart contracts and IoT technologies.

At the time of launch, the platform accounted for about $15 \%$ of the market, now it is 35\%. Among TradeLens partners (users) you can find 15 of the largest shipping carriers, including Seaboard Marine, KMTC, ZIM, Safmarine, Boluda, Sealand, Namsung and APL.

In collaboration with MIT, Toyota [5] began recruiting a number of partners who specialize in various aspects of Blockchain technology so that they can explore how this technology could be applied to the automotive industry.

Toyota has already unveiled a number of projects that it hopes will help allay human concerns about handing over control to an autonomous vehicle, or "third space," as they now call it, for example Yui, its artificial intelligence (AI) satellite. It is now clear that they want to go further, which means developing a platform that can track and disseminate information about the safety of individual vehicles, how their owners use them, and reduce fraud.

"Building safe and reliable autonomous vehicles may require hundreds of billions of miles of human driving data," says Chris Ballinger, director of mobility services and chief financial officer at Toyota Research Institute. and manufacturers are shortening the time to reach this goal, thereby increasing the safety, efficiency and usability of autonomous driving technology. "

The purpose of this work is to implement tracking of car actions using a modified blockchain platform, namely, a safe and reliable system for sending the parameters of the current state of each car through the neighboring ones. In our system, vehicles confirm messages from neighboring vehicles within a radius of 100 - 150 meters. Messages are signed by the senders and every neighboring vehicle equipped with such a system.

\section{Intelligent Transport Systems Trends}

Below are examples of intelligent systems embedded in modern vehicles.

Xiu-feng Chen [13], when analyzing improved transport in China, argues that vehicles equipped with ITS technology are able to predict well any hazardous situations on the vehicle in advance, and therefore reduce reaction times, which leads to improved road safety and leads to a decrease in the number of accidents in transport.

In Italy, Benza [13] et al. Argue that in these areas of freight management, ITS improves the management of freight vehicles and vehicle parking, and provides information on weather and other critical traffic situations.

Making the most of ITS implementation methods is showing increased potential in the following areas: raising driver awareness of the current road situation, road safety and precautions, which will simultaneously have an impact on good transport management. 
ITS solutions and applications are wide and varied. One of the most common vehicle ITS is the airbag, which accumulates information just before a collision.

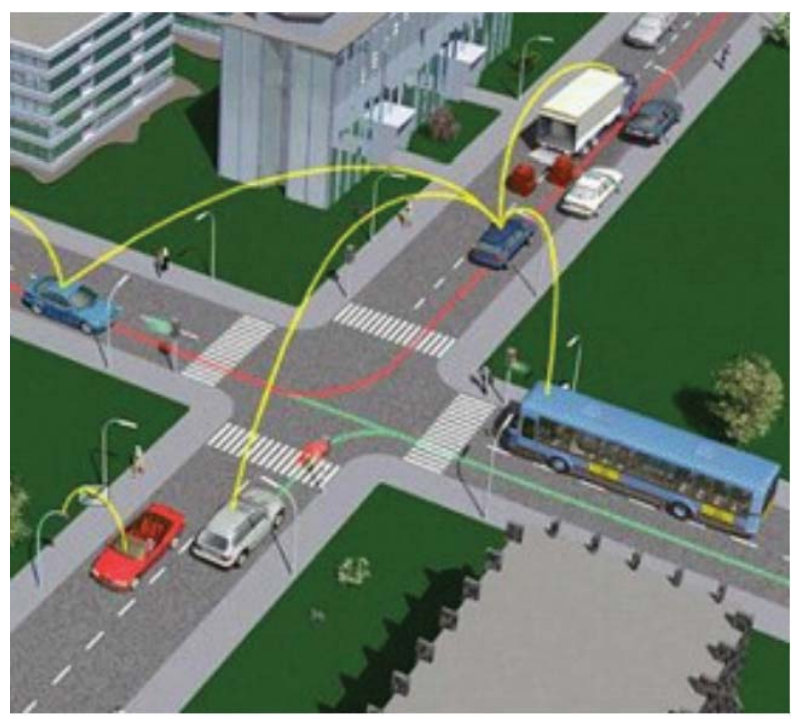

Figure 1. Application of ITS in transport [13]

One aspect is related to the introduction and adaptation of advanced technologies within the vehicle boundaries to communicate with other moving vehicles, as well as with the vehicle environment, which will help the driver to better and more accurately assess his driving situation.

McDonald [13] argues that this advanced driver assistance system was initiated to create improved technology integration using sensor and communication expertise so that the vehicle driver can maneuver more appropriately to avoid collisions, complex road network negotiations, and it is safer to follow other vehicles.

Collision Notification and Collision Avoidance is an application of intelligent vehicle technology that is designed and engineered to detect and communicate the magnitude as well as the exact location of incidents to agencies and services responsible for coordinating appropriate emergency response actions in the shortest possible time.

The following collision rates are used:

- $10 \mathrm{~Hz}$ for vehicle emergency warning, intersection collision warning and collision risk warning.

- $2 \mathrm{~Hz}$ for slow vehicle indication, motorcycle approach indication and optimal traffic light speed.

- $1 \mathrm{~Hz}$ to $10 \mathrm{~Hz}$ for speed limit notification.

Collision Warning and Collision Avoidance: It uses an intelligent technology system to warn the vehicle driver of an unfavorable approach to a nearby vehicle through the use of sensor radars, audio alerts and video on the screen.

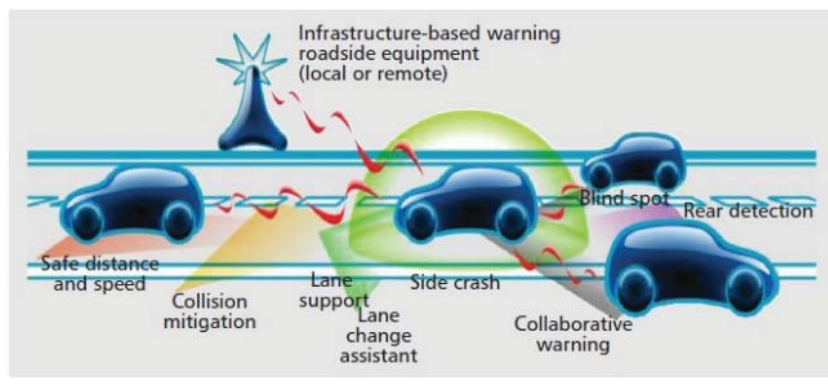

Figure 2. An example of communication between vehicles [13]

Logi and Ritchie [14] described a real-time knowledge-based system (KBS) to support decisionmaking in the selection of integrated traffic management plans after non-recurring congestion. In this study, two algorithms were developed - an algorithm for combining data for congestion analysis and an algorithm for selecting control plans. The test results showed that the use of Traffic Congestion Management (TCM) reduced travel times from $1.9 \%$ to $29.0 \%$, and the average stopping speed decreased from $14.8 \%$ to $55.9 \%$.

Fagri and Hamad [14] studied the use of GPS in traffic management. In their study, GPS applications were involved in collecting traffic data such as travel time, speed and delay on 64 major roads in Delaware. When comparing the mean and variance of the results obtained by both methods, no significant differences were observed. GPS data was found to be $50 \%$ more efficient in terms of labor force.

Hernandez et al. [14] incorporated the use of artificial intelligence techniques in traffic management and presented a multi-agent architecture for intelligent traffic management systems. To support real-time traffic management decisions, two knowledge-based multi-agent systems, InTRYS and TRYSA2, have been developed. The performance of both systems was evaluated and the general applicability of multi-agent architectures for intelligent traffic management was given.

Zhenlin et al. [14] studied the effectiveness of the Beijing Intelligent Traffic Management System (ITMS). In this study, urban transport systems, socio-economic system and energy environment system were adopted as the input system, and the efficiency of traffic management and urban transport as the output system. Field data for Beijing from 2000 to 2010 are used for empirical analysis. The results of the study showed that ITS improved the overall efficiency of Beijing transportation.

\section{Blockchain technology in terms of applicability in transport systems}

The provenance, immutability, transparency and security of data are becoming integral parts of the Blockchain and all industries are trying to investigate this. One such industry where blockchain can prove useful is the transportation industry. 
When we talk about the transportation industry, you should know that it is an integral part of the supply chain. In fact, transport is the foundation for all industries. Thus, it becomes imperative that companies have a seamless supply chain. Hence, it is essential that the transport industry runs smoothly.

Before understanding how Blockchain can revolutionize the transport industry, it is important to understand the challenges this sector is facing. From dispute resolution to administrative efficiency and order tracking, Blockchain has a solution to the problems that have plagued the transportation industry for decades. Let's take a look at some of these issues.1.

\section{Tracking}

One of the main challenges facing the transport industry is payment and dispute resolution. Did you know that about $\$ 140$ billion is tied to transportation dispute settlement payments every day? It is time consuming and in many cases the transport industry depends on a third party, but with Blockchain there is no need for a third party. With an efficient Blockchain tracking system, we can easily track vehicles and know their status.

\section{Transportation of temperature controlled products}

The problem arises with temperature controlled goods, with approximately $8.5 \%$ of sensitive pharmaceutical shipments reportedly experiencing temperature swings. This adversely affects the shipment of such products as they exceed their specified temperature specifications. This results in loss of travel costs. Let's see how Blockchain can solve this problem.

Using Blockchain to authenticate data, IBM is partnering with other global companies to launch a platform that can guarantee the required efficiency, authenticity and transparency in the supply chain. When we talk about temperature controlled products, transportation depends on on-time delivery, and this is increasing with Blockchain.

\section{Smart contracts}

This is another area where we can find blockchain applications in the transportation industry. Having a smart contract ensures how and when the product is delivered, the smart contract is executed, and payment begins. Smart contracts are one of the best Blockchain technology applications. It is a preprogrammed contract that defines the terms and conditions. These conditions are predefined, after these conditions are met, payment is made.

\section{Tracking loading and unloading}

This is another area of application for blockchain. Similar to record tracking, Blockchain can be helpful in validating a driver record. This becomes beneficial when the freight broker is trying to achieve the load capacity at a specific location. If the broker identifies a new carrier, he can use the Blockchain ledger to verify the carrier and assign the load. With Blockchain technology, we can have a decentralized system in which all media records across the entire industry can be stored.

\section{It makes the loading on board more reliable}

One of the common problems faced by the transport industry is the cargo platform. In a traditional system, it is possible that the data can be changed and therefore create the wrong load. With Blockchain, shippers can easily submit time stamped shipments that are verified by the Blockchain network. These data are unchanged.

\section{IoT for communication with the car}

Many companies are implementing V2V or vehicleto-vehicle communication. This system allows various cargo vehicles to communicate, thus providing fuel savings. This data, when stored in the Blockchain, can help transport companies optimize their operations.

Exonum is a platform that allows you to create decentralized, secure and reliable applications on the blockchain. The platform is designed for companies, organizations and even governments. Using the Exonum solution, these organizations can create their own private network that meets the needs of a particular company and provides unprecedented security by integrating the project with the blockchain.

Exonum is the fastest private blockchain that can process up to 9000 transactions per second. Programs for the Exonum blockchain platform are written in Rust, which Bitfury experts call the safest programming language, guaranteeing no memory management problems. A Rust program compiles directly to machine code, so it runs faster than virtual bytecode.

At the moment, the Exonum platform is used for pilot projects in the state registries of three countries: Georgia, Ukraine, Russia.

This largely applies to the management of large systems, to which the transport belongs. Failure to follow simple rules and recommendations can lead to serious consequences, including causing a traffic collapse.

\section{Basic system architecture}

Figure 3 shows the basic diagram of the proposed vehicle tracking system using blockchain technology.

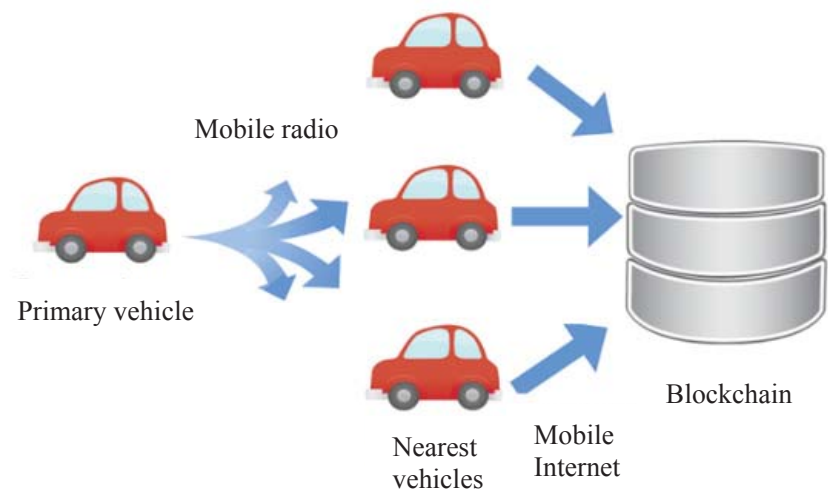

Figure 3. System architecture

Sending data to the blockchain works in three stages as follows: 
1. The main vehicle determines its position using any global positioning system, draws up a message containing the following information:

- his identification number

- its geographic coordinates

- exact current time and date

- message signature (the signature key is issued by the registering authority).

2. The main vehicle sends this data through a short distance transceiver module similar to the NRF24L01. It should send wireless signals at a distance of up to 150 meters so that only those vehicles that are nearby (nearby vehicles) are involved in the communication process $[2,3]$.

3. Each adjacent vehicle receives a signal and composes a new message that includes the following information:

- his identification number

- its geographic coordinates

- full unmodified copy of the original message

Please note that it is not necessary to include the current date and time on adjacent vehicles. They are automatically recorded by the back-end system (blockchain). Although it is important that the main vehicle turns it on, this is important for safety reasons.

We assume that the terminals use the following equipment:

1. Some simple PC boards like Arduino or Rasberry Pi.

2. $3 \mathrm{G} / 4 \mathrm{G}$ mobile internet module that supports sending messages to a SIM card with a request to sign it.

3. A transceiver like NRF24L01 must operate with the same protocol and cover at least a 150 meter radius area.

4. Any global positioning system that provides accurate geographic coordinates.

\section{System security}

Although we use the Blockchain system (Exonum) to ensure that no one can add any information backdated, and no one can edit any information that is already stored, there are several other security issues that we need to take care of [4].

Case 1. The nearest car is trying to spoof the received message.

He may try to change the vehicle ID, position or time. In this case, the signature of the main vehicle will not match. An attempt to forge a signature takes too long, the system checks if the difference between the time in the message and the current system time does not exceed 1 minute. At the same time, 1 minute is a fairly large interval that will allow you to have some inaccurate timing systems.

A nearby vehicle may keep the sequence of messages in some position and try to fake the same position later. This would not be possible since the date and time stamp in the messages is also signed by the main vehicle. This is why it is important to include it in the message of the main vehicle, rather than include it in the messages of neighboring vehicles.

Case 2. The main vehicle is trying to spoof its ID, time, or location.

The problem of time has already been considered in the previous case. Faking an id is not easy, as you need to have a vehicle signature and it is securely stored inside the SIM card.

So, we need to consider the problem of spoofing its location. If the main vehicle sends a different location, neighboring vehicles shouldn't worry about it, they still send the message further into the blockchain [5-7]. But nearby vehicles are also sending their own locations, so the system can detect that the distance between the two vehicles is too great (more than $1 \mathrm{~km}$ ) and flag it as a potential scam. $1 \mathrm{~km}$ should be enough for a SIM card to sign a message.

Case 3. Both the main vehicle and nearby vehicles try to fake the location of the main vehicle.

Consider a case where several vehicles are hacked and they are trying to fake the location of one of them (the main vehicle). This scenario is possible and the system can save all the data exactly as it was sent.

Having a full track with the date and time stamp of the main vehicle, it is easy to check other vehicles that have declared their position at the same time and in the same position up to 150 meters. It would also be suspicious that all messages from the main vehicle are corroborated by the same nearby vehicles [8].

Unfortunately, if there were no other vehicles in the path of the vehicle, it is very difficult to verify with a $100 \%$ certainty whether the case has been tampered with. Although, verification of this case is still possible: since all data is sent via the mobile Internet. Thus, having the IP addresses of nearby vehicles, it is possible to query the communication provider which stations were used by this client.

\section{Conclusion}

This work will serve as an important step towards the development of traffic control systems for connected and autonomous vehicles, contributing to the solution of the problem of cybersecurity, as well as in the investigation of various types of crimes.

In this paper, the cases of the presence of some fixed terminals were considered, which do not necessarily use a real global positioning system and mobile Internet connection, but are installed by the system supplier to act as nearby vehicles along some roads in the city or on highways at some intervals [4- five]. These clients can help control vehicles with $100 \%$ reliability. Even a single message signed by the terminal would have been enough to assert that the car was passing by.

The paper considers a system that is safe and allows you to register the movement of a large number of vehicles. Given your location data at any given time, it is relatively easy to calculate the average speed as well as the speed at a given time. If the car is used for some 
criminal purpose, this system will help us calculate it. Of course, in the event of a violation of the law, attackers can disable the internal warning system, then a car with the system turned off is already an object of special attention and will be suspicious for entire system. Also, in order to prove that the vehicle did not take part in any offense, it is enough to check the system logs, this will be reliable proof of innocence.

\section{References}

1. Kohei Arai, Rahul Bhatia Editors. Advances in Information and Communication: Proceedings of the 2019 Future of Information and Communication Conference. Vol. 1
2.
Logistica
na
blockcheine.
https://

http://www.comnews.ru/content/121845/2019-09-06/logistikana-blokcheyne/ Retrieved: Oct, 2019

3. Tehnologiya blockchain $\mathrm{v}$ transportnoi logistike https://crypto-fox.ru/article/p2p-kreditovanie. Retrieved: Apr, 2018

4. Maersk, IBM launch first blockchain joint venture for trade, transportation http://www.dcvelocity.com/articles/ 20160116-maersk-ibm-launch-first-blockchain-joint-venturefor-trade-transportation. Retrieved: Jan, 2018

5. Toyota ties up with blockchain startups to connect its world. https://www.311institute.com/toyota-ties-up-withblockchain-startups-to-connect-its-world. Retrieved: Jun, 2017.
6. M. Svon Blokchein. Shema novoi ekonomiki. Moscow: Olimp-biznes, 2016

7. https://www.forbes.ru/tehnologii/360953-umnyemashiny-kak-iskusstvennyy-intellekt-menyaet-avtorynok.

8. Kurt Dresner, Peter Stone. A Multiagent Approach to Autonomous Intersection Management, Journal of Artificial Intelligence Research 31. 2008. P. 591-656.

9. K. Dresner. Autonomous Intersection Management. $\mathrm{PhD}$ thesis, The University of Texas at Austin, 2009.

10. O.N. Vasilenko Teoretiko-chislovye algoritmy v kriptografii. Moscow: MCNMO, 2003. 328 p. ISBN 5-94057103-4.

11. Chto takoe blokchein [Elecrtonnyi resurs] - Elekton. tekstovye dan. - rezhim dostupa https://habrahabr.ru/company/ /blog/329276/, svobodnyi.

12. K. Dresner and P. Stone. A multiagent approach to autonomous intersection management. Journal of Artificial Intelligence Research (JAIR), March 2008.

13. Nkoro, Anne \& Vershinin, Yuri. (2014). Current and future trends in applications of Intelligent Transport Systems on cars and infrastructure. 2014 17th IEEE International Conference on Intelligent Transportation Systems, ITSC 2014. 514-519. 10.1109/ITSC.2014.6957741.

14. SINGH, Bhupendra and GUPTA, Ankit. Recent trends in intelligent transportation systems: a review. J. Transp. Lit. [online]. 2015. Vol. 9. No.2. P. 30-34. ISSN 2238-1031. http://dx.doi.org/10.1590/2238-1031.jtl.v9n2a6. 